Results: Tc 99 m tilmanocept localised most effectively at the $200 \mu \mathrm{g}$ mass dose 2-3 hours post- administration in RA subjects (cohort 4). Localization was observed in $60 \%$ of the subjects in joints of the bilateral wrists, hands, and knees. No localization was observed in $\mathrm{HCs}$ receiving the same mass dose (cohort 2).

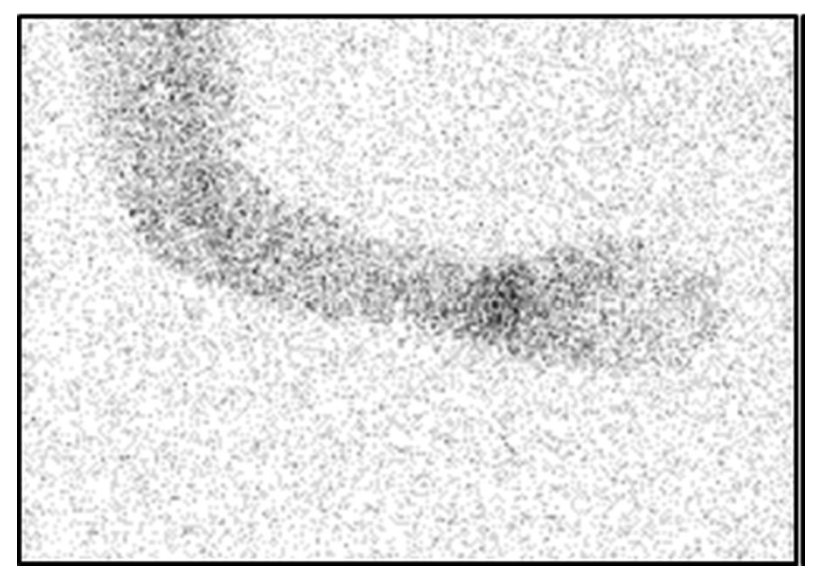

Abstract AB0248 - Figure 1. Contrast of wrist and elbow of SC RA subject

Conclusions: Our findings represent a potential advance in the imaging of RA using a novel immunodiagnostic imaging strategy to specifically visualise macrophage-mediated elements of the RA disease process. This study demonstrates the potential for Tc $99 \mathrm{~m}$ tilmanocept to be used as an imaging tool for macrophage-mediated synovial inflammation in RA patients. This strategy can be used to identify patients at risk for activated macrophage-mediated joint damage, to quantify synovitis and disease activity, to provide further insight into immunemediated mechanisms of

RA, and to enable future targeted delivery of immunomodulatory therapeutics.

Acknowledgements: Navidea Biopharmaceuticals

Russell Engleman Rheumatology Research Centre, University of California San Francisco

Disclosure of Interest: J. Graf Grant/research support from: Navidea Biopharmacueuticals, B. Abbruzzese: None declared, F. Cope: None declared, S. Behr: None declared, J. Sanders: None declared, A. Kissling: None declared, D. Ralph: None declared, J. Shuping: None declared, M. Blue: None declared, C. Hartings: None declared, R. Hershey: None declared, H. Bailey: None declared, A. spaulding: None declared, M. haynam: None declared, A. Ismail: None declared DOI: 10.1136/annrheumdis-2018-eular.7533

\section{AB0249 INFLUENCE OF AUTOANTIBODIES PROFILE ON DISEASE ACTIVITY MEASUREMENT IN A COLOMBIAN COHORT OF RHEUMATOID ARTHRITIS PATIENTS}

J.B. Florez, G. Quintana, P. Mendez, P. Coral, on behalf of Reumavance Group. Department of Rheumatology, Fundacion Santa Fe de Bogotá University Hospital, Bogotá, Colombia. Rheumatology, Fundacion Santa Fe de Bogota, Bogotá DC, Colombia

Background: Traditionally, the role of Rheumatoid Factor (RF) and/or Anti-citrullinated antibodies (ACCP) presence have characterised for diagnosis and prog nosis of Rheumatoid Arthritis (RA). However, Anti-nuclear antibodies (ANA) are not routinely measured for the diagnosis of the disease or RA prognosis establishment during first appointment. Recently, evidence showed that positive ANA titles could be considered as poor prognosis factor for RA, and also a higher probability of developing immunogenicity against biologic therapies.
Objectives: To compare the disease activity measurement from a Colombian cohort of patients with RA, based on their auto-antibodies profile.

Methods: The study used a cohort of Colombian patients with RA. A database was developed using the information from the clinical records. The data included were: RF, ACCP, ANA, and the disease activity measured using DAS-28 ESR. Disease activity results were obtained in the following periods of time: $0,3,12,24$ and 36 months. Patients were classified based on the different autoantibody pro files (RF/ACCP/ANA:- , +-, -+-, -+, ++-, +-+, -++, +++). Mean DAS-28 ESR results from each period of time were calculated. Also mean weekly Methotrexate (MTX) dose was calculated for each profile. Mean differences between initial, and the follow-up period were calculated using Kruskal-Wallis test. Statistical analysis was made using STATA 12.0 software.

Results: 635 patients with RA were included. $32 \%$ of them were men, and $68 \%$ were women. Mean age was 54.3 years. The most prevalent profile was +++ with 118 patients, and the less frequent was +-. Patients with +++profile had the best response to treatment over time, but also they required more MTX dose. Less response during time was observed with +- profile, however the amount of patients from these group was relatively low. As it was expected,- - profile patients required less weekly MTX dose $(9.26 \mathrm{mg})$. It was interesting that patients with +profile present a worst outcome based on DAS-28 activity, and less response to the treatment.

Conclusions: Results from the study suggest the importance of including the measurement of ANA titles in the initial categorization and follow-up of patients with RA. The presence of ANA seems to have a worst prognosis. ANA co-exis tence with ACCP appear to have a worst outcome, compared to ++- or +--profile. Auto-antibody profile in RA could direct the best therapeutic strategy for each patient. Validation of these results are required based on other cohorts.

\section{REFERENCE:}

[1] Sanmarti R, Gomez-Puerta JA. Biomarcadores en la artritis reumatoide Reumatol Clin. 2011;6(S3):S25-S28.

Disclosure of Interest: None declared

DOI: 10.1136/annrheumdis-2018-eular.7589

\section{AB0250 ENDOTYPING OF ARTHRITIC PATIENTS USING NOVEL SEROLOGICAL BIOMARKERS}

J.P.M. Blair ${ }^{1}$, C. Bager ${ }^{1}$, M. Weinblatt ${ }^{2}$, M. Karsdal ${ }^{1}$, A. Platt ${ }^{3}$, A.-C. Bay-Jensen ${ }^{1}$ ${ }^{1}$ Rheumatology, Nordic Bioscience, Copenhagen, Denmark; ${ }^{2} 2$ Division of Rheumatology, Immunology and Allergy, Brigham and Women's Hospital, Boston, Massachusetts; ${ }^{3}$ AstraZeneca, London, UK

Background: Accurate patient stratification is critical if medical professionals are to adopt a precision medicine approach when planning clinical trials or prescribing medication. This approach results in a superior level of drug response in the target group, a reduction in adverse effects and reduced costs for payers.

Best practice treatment recommendations and disease activity markers such as DAS28, as opposed to a treat to target approach, guide current treatment of arthritic disease. There is currently a lack of tools to enable patient stratification, in part due to traditional biomarkers reflecting systemic inflammation rather than the target tissue.

Objectives: In this paper, we explore the use of a combination of novel tissue specific biomarkers for patient clustering with the objective of identifying different disease profiles.

Methods: Four biomarker substudy cohorts were pooled for this study, including two RA studies; LITHE $(n=574)$ and OSKIRA-1 $(n=131)$ and two OA studies; SMC1 $(n=447)$ and SMC2 $(n=81)$ all of which have been described in detail before. Whilst the principle focus was to examine RA patient profiles, OA studies were included to enrich the cohort with a non-RA population. OSKIRA and LITHE both had measurements at 24 weeks with additional measurements at 52 week s in LITHE.

\begin{tabular}{|c|c|c|c|c|c|c|c|c|c|c|}
\hline $\begin{array}{l}\text { PROFILE } \\
\text { (FR/ACCP/ } \\
\text { ANA) }\end{array}$ & $\begin{array}{l}\text { DAS-28 } \\
\text { (INITIAL }\end{array}$ & $\begin{array}{c}\text { DAS-28 } \\
\text { (3 MONTHS) }\end{array}$ & $\mathrm{N}$ & $\begin{array}{c}\text { DAS-28 } \\
\text { (12 MONTHS) }\end{array}$ & $\mathbf{N}$ & $\begin{array}{c}\text { DAS-28 } \\
\text { (24 MONTHS) }\end{array}$ & $\mathbf{N}$ & $\begin{array}{c}\text { DAS-28 } \\
\text { (36 MONTHS) }\end{array}$ & $\mathbf{N}$ & $\begin{array}{l}\text { MEAN WEEKLY MTX DOSE } \\
(\mathrm{mg})\end{array}$ \\
\hline- & 3.317 & .3072603 & 73 & 0.1267188 & 64 & $0.9367073^{*}$ & 41 & $0.8593334^{*}$ & 30 & 9.26 \\
\hline+- & 3.375 & -0.2905715 & 7 & -0.5806667 & 6 & -0.2648572 & 7 & 0.17 & 4 & 10.82 \\
\hline -+- & 3.434 & .263663 & 92 & $0.6267033^{*}$ & 91 & $0.7705405^{\star}$ & 74 & $0.6138596^{*}$ & 57 & 14.01 \\
\hline-+ & 3.201 & .0314815 & 27 & -0.2926087 & 23 & 0.1705263 & 19 & 0.8038461 & 13 & 10.2 \\
\hline++ & 4.123 & $.8346712^{\star \star}$ & 73 & $1.048^{\star *}$ & 66 & $1.23^{\star *}$ & 51 & 0.53 & 38 & 16.19 \\
\hline-++ & 3.542 & .3822857 & 56 & $0.5656604^{*}$ & 53 & $0.71325^{*}$ & 40 & $0.8733333^{*}$ & 33 & 14.91 \\
\hline+-+ & 4.695 & $1.57^{*}$ & 12 & $1.77^{*}$ & 14 & 3.359 & 6 & 2.03 & 2 & 15.69 \\
\hline+++ & 4.118 & $.6474576^{*}$ & 118 & $0.9343478^{* *}$ & 115 & $1.196^{* \star}$ & 86 & $1.25^{\text {** }}$ & 66 & 15.83 \\
\hline
\end{tabular}

